\title{
Cultes liturgiques et dévotions populaires dans les comtés de Portneuf et du Lac-Saint-Jean
}

Jean Simard

Volume 43, 1976

URI : https://id.erudit.org/iderudit/1007226ar

DOI : https://doi.org/10.7202/1007226ar

Aller au sommaire du numéro

Éditeur(s)

Les Éditions Historia Ecclesiæ Catholicæ Canadensis Inc.

ISSN

0318-6172 (imprimé)

1927-7067 (numérique)

Découvrir la revue

Citer cet article

Simard, J. (1976). Cultes liturgiques et dévotions populaires dans les comtés de Portneuf et du Lac-Saint-Jean. Sessions d'étude - Société canadienne d'histoire de l'Église catholique, 43, 5-14. https://doi.org/10.7202/1007226ar

Tous droits réservés @ Les Éditions Historia Ecclesiæ Catholicæ Canadensis Inc., 1977
Ce document est protégé par la loi sur le droit d'auteur. L'utilisation des services d'Érudit (y compris la reproduction) est assujettie à sa politique d'utilisation que vous pouvez consulter en ligne.

https://apropos.erudit.org/fr/usagers/politique-dutilisation/ 


\section{Cultes liturgiques et dévotions populaires dans les comtés de Portneuf et du Lac-Saint-Jean}

Après quarante ans d'enquêtes sur le culte des saints qui l'avaient conduit dans 542 des 630 communes de la Savoie, l'ethnologue Arnold Van Gennep concluait à l'indépendance de deux formes de cultes, les uns liturgiques, les autres populaires, en ajoutant "qu'il y a parfois, rarement, des coincidences ou des points de jonction ${ }^{1} »$.

Qu'en est-il au Québec? Doit-on penser qu'il en va ici comme là-bas ou que les rapports particulièrement étroits qu'a toujours entretenus le peuple du Québec avec son clergé interdisent de croire à l'existence de cultes parallèles ? C'est dans le cadre de cette interrogation qu'ont été élaborés, depuis quelques années, des enquêtes et des travaux dont nous présentons maintenant, Paul Jacob et moi-même, quelques résultats.

\section{Méthodologie}

Les conclusions de Van Gennep s'appuyaient sur l'exploitation de deux types de documents, les uns dits liturgiques: répertoires de vies de saints, recueils iconographiques, dictionnaires et calendriers liturgiques, martyrologes, bréviaires, catéchisme, missels, sacramentaires, registres financiers des diocèses, archives administratives des paroisses, relevés toponymiques des paroisses et autres lieux de cultes liturgiques ${ }^{2}$; les autres dits populaires: petits oratoires non consacrés et devant lesquels les fidèles prient sans

1 A. Van GenNEP, "Cultes liturgiques et cultes populaires» (paru en 1934 dans le Folklore brabançon), Culte populaire des saints en Savoie, Archives d'ethnologie française, $n^{0}$ 3, Paris, G.-P. Maisonneuve et Larose, 1973, 157-167.

2 Cette catégorie de documents, pour le Québec, a été récemment analysée avec beaucoup de prudence par Pierre SAVARD, "Sur les noms de paroisses au Québec, des origines à 1925 », Sessions d'étude de la Société canadienne d'Histoire de l'Église catholique, 1974, 105-113. 
qu'il n'y soit dit de messe, ni périodique ni même occasionnelle, surtout les enquêtes directes auprès de milliers de Savoyards, recrutés dans toutes les parties du petit pays et pratiquant tous les genres de la vie rurale.

On comprendra tout d'abord que la distinction établie entre ces deux types de documents repose sur l'idée qu'il existe deux types de productions culturelles dans les sociétés traditionnelles européennes, l'une de l'élite, qui est écrite et conservée, l'autre populaire, qui est surtout orale et matérielle, et dispersée. En second lieu, le fait d'admettre l'existence distincte d'archives de l'élite et d'archives propres au peuple s'appuie essentiellement sur la reconnaissance de rapports dialectiques de groupes au sein d'une même société, mais ici de groupes culturels plutôt que sociaux ${ }^{3}$. Ici surgissent alors les concepts de comportements ou de conduites et de mentalités qui n'ont que peu à voir avec les stratifications sociales et économiques. Entre le moment où le curé d'une paroisse rurale expliquait aux enfants les vérités contenues dans le catéchisme, par exemple sur l'unité et la trinité divine, et celui où il distribuait les bonbons et faisait exploser les feux d'artifice au pied de la croix de chemin ${ }^{4}$, il y a passage instantané du groupe culturel qui lui fournit ses modèles à celui dont il tire ses sources. C'est donc sur cette distinction fondamentale qui existe, dans les sociétés complexes telles que la France et le Québec, entre culture savante, dirigeante et pourvoyeuse de modèles, et culture populaire, dirigée, plus portée vers la tradition que vers l'innovation,

3 C'est dans cette distinction que P. Saintyves a fondé sa notion de «mentalité populaire» (Manuel de folklore, Paris, E. Nourry, 1936, 33.). Même si les traits caractéristiques de cette mentalité dite populaire se retrouvent normalement et le plus souvent chez les non instruits, les économiquement faibles, l'expérience démontre que ces traits persistent souvent dans les conduites de ceux qui détiennent le pouvoir, surtout quand les détenteurs de ce pouvoir sont eux-mêmes issus du peuple. Cette façon de voir les choses permet aussi d'avancer plus vite dans l'étude de la vie populaire puisqu'il n'est plus besoin d'attendre les résultats d'analyse des groupes sociaux, de leur composition, de leurs origines, de leurs caractéristiques socio-professionnelles etc... En cela, Saintyves a été suivi par A. Varagnac (Civilisation traditionnelle et genres de vie, Paris, Albin Michel, 1948.), et, plus récemment, par Jean CUISENIER (L'Art populaire en France, Fribourg, Office du livre, 1975, 36.).

4 Voir, dans le présent volume, l'article de Paul Jacob. 
que doit en principe reposer celle qui est établie par Van Gennep entre les types de productions et les documents eux-mêmes.

Pour reprendre un peu cette démarche théorique et méthodologique, il fallait, dans un premier temps, dégager de la production culturelle québécoise des séries documentaires qui étaient assez homogènes et identifiables spontanément aux groupes culturels populaires; faire de même dans les groupes culturels savants ou officiels. Dans un deuxième temps, il fallait s'assurer que chacune de ces documentations pouvait être "sémantisée " par ceux-là même qui les avaient produites. Troisièmement enfin, procéder à la confrontation de ces discours, puis les évaluer du point de vue de la question posée au point de départ.

La première expérience a donc été tentée dans les comtés de Portneuf et du Lac-Saint-Jean. Et les deux séries documentaires retenues ont été tout d'abord les monographies paroissiales, émanation évidente de la culture historique et théologique, donc savante et officialisante, puisque leurs auteurs sont des lettrés, en majorité des ecclésiastiques. En second lieu, ce furent les croix de chemins de ces deux comtés, spécialement les saints habitants de leurs niches, qui ont été retenues comme témoins de la culture populaire. Les monographies devaient en principe renseigner sur les cultes liturgiques, proposés ou pratiqués dans ces régions, tandis que le contenu des niches de croix de chemins devait faire connaître les tendances des dévotions pratiquées dans le peuple. Dans ce dernier cas, les enquêtes exhaustives auprès des propriétaires, parfois des constructeurs, ont démontré avec netteté que ces modestes monuments ont été érigés en très grande majorité par des cultivateurs, qui voyaient là le moyen le plus sûr de conjurer les fléaux naturels qui menaçaient presque annuellement leurs récoltes. Peu de place ici aux immixtions de l'autorité religieuse, sauf parfois pour la bénédiction. Au contraire, les rares interventions officielles ont bien montré que l'Église ne se reconnaissait pas entièrement dans ces croix chargées des instruments de la passion: " La croix, écrivait en $1818 \mathrm{M}^{\mathrm{gr}}$ Plessis, l'évêque de Québec, est assez respectable par elle-même et doit suffire à la dévotion des fidèles, surtout si elle porte un Christ bien travaillé » 5 .

5 Cité par L. DésY et J.R. PoRTer, Calvaires et croix de chemins du Québec, Montréal, Hurtubise HMH, 1973, 56. 
Le choix des comtés municipaux de Portneuf et du LacSaint-Jean ${ }^{6}$ trouve sa justification dans le fait qu'en 1972, ces deux terrains ethnographiques ne furent ni plus ni moins que ratissés ${ }^{7}$, et qu'on a trouvé dans ces deux comtés un nombre à peu près égal de croix, ce qui a permis, l'année suivante, dans le cadre d'un séminaire d'études avancées que j'animais ${ }^{8}$, de dresser des tableaux comparatifs et d'esquisser des hypothèses en vue de déterminer quelque peu le paysage religieux de ces régions. Quant aux monographies paroissiales, elles sont aussi en nombre sensiblement égal ${ }^{9}$ et elles off rent le grand avantage, non seulement de constituer un genre littéraire bien homogène, une sorte de dépôt d'archives de la culture savante, mais aussi de "sémantiser " les objets qu'elles présentent aux lecteurs. Van Gennep avait abordé aussi cette documentation qu'il conseillait d'utiliser avec la plus grande prudence :

Toutes les fois qu'on lira dans une monographie régionale des formules comme "nos populations ont eu la plus grande vénération " pour tel saint, ou tel autre, on fera bien de chercher sur quels documents l'auteur appuie son affirmation; le

6 Les comtés municipaux, que l'on doit bien distinguer des circonscriptions électorales, de Lac-Saint-Jean Est et de Lac-Saint-Jean Ouest ont été ici regroupés, d'abord parce qu'ils représentent totalement la région ou le «pays folklorique» du Lac Saint-Jean, et ensuite parce qu'ils fournissaient, au chapitre des croix, une base de comparaison équivalente à Portneuf. Notre outil de travail a été le Répertoire des municipalités, Québec, Ministère de l'Industrie et du Commerce, Bureau de la statistique du Québec, 1970.

7 Le Lac-Saint-Jean, par Paul Carpentier, au compte du Musée national de l'Homme, Ottawa; Portneuf, par Bernard Genest, qui était alors mon assistant grâce à une subvention du Conseil des Arts du Canada.

8 Régis JEAN, Étude du contenu des niches des croix de chemins en rapport avec les dévotions populaires régionales dans les comtés de Portneuf et du Lac-Saint-Jean, manuscrit dactylographié, Québec, C.E.L.A.T., Université Laval, 30 janvier $1974,30 \mathrm{p}$.

9 Ont servi de guides les ouvrages suivants: Antoine Roy, Les histoires de paroisses, (Québec), Archives nationales du Québec, (194-), 255-364; André Beaulieu et William F.E. Morley, La Province de Québec, (Toronto), University of Toronto Press, (1971). Bien que A. Roy compile 9 monographies pour Portneuf et 4 pour le Lac-Saint-Jean, tandis que Beaulieu et Morley n'en compilent que 4 pour Portneuf et 12 pour le LacSaint-Jean, seules les histoires de paroisse proprement dites ont été retenues. Les études de géographie, telles celles de Raoul Blanchard, les nombreuses publications de la fin du $19^{\text {e }}$ siècle, commanditées par les ministères, ne nous ont pas servi. 
plus souvent on constatera que c'est sur des documents liturgiques, sur des listes de toponymes ou de patronages, dans le meilleur cas, sur des listes de chapelles; et l'on fera bien de rechercher pour chaque cas particulier la concomitante populaire nécessaire 10.

Il faut enfin dire un mot d'une certaine disparité dans la cueillette des données. Dans le comté de Portneuf, B. Genest utilisait un Questionnaire de l'enquêteur ${ }^{11}$ que j'avais mis au point dès 1971 et qui, malgré certaines lacunes, notamment sous la rubrique des types iconographiques des statuettes de niches, comportait l'avantage de fournir des entrées uniformes. Dans le cas du Lac-Saint-Jean, P. Carpentier avait procédé autrement. Le carnet d'enquête était son principal outil et les faits recueillis, bien que toujours très précis et très éclairants, manquaient un peu de cette uniformité qui est éminemment souhaitable lorsqu'il s'agit ensuite de compiler en vue de la comparaison. Ce ne sont là toutefois que des lacunes qui, je crois, n'ont pas joué sérieusement quand est venu le moment d'établir et d'interpréter ces comparaisons.

\section{Les données}

Au moment de l'inventaire, le comté de Portneuf comptait 80 croix de chemins ${ }^{12}$. Sur ce nombre, 29 comportaient des niches " habitées » de statuettes, ou qui l'avaient été dans un passé récent. $\mathrm{Au}$ Lac-Saint-Jean, on a dénombré 83 croix, dont 28 qui étaient

10 Van GenneP, loco cit., 165.

11 Ce questionnaire, de type fermé et qui comporte 773 cotes décimales, dont deux grilles qui comptent à elles seules 492 de ces 773 cotes, est uniquement destiné à décrire l'artefact. Il ne prévoit cependant pas tout, et dans le cas des types iconographiques des statuettes, il n'en fournit explicitement que sept : la Vierge, la Vierge et l'Enfant, le Christ au Sacré-Caur, la Sainte-Famille, Thérèse de l'Enfant-Jésus, l'Enfant de Prague, la Descente de croix. Pour tous les autres cas, il y a une cote ouverte et les enquêteurs utilisent nécessairement un vocabulaire différent ou encore le même enquêteur, comme nous le constaterons plus loin, modifie le champ sémantique de son vocabulaire au cours d'une même journée.

12 La croix de chemin se définit par sa fonction : elle est destinée aux usagers des chemins, pour leurs pratiques de piété. Il s'agit normalement de la communauté locale qui y fait les exercices du mois de Marie, ou du simple passant qui adresse à la croix quelque marque de respect. 
habitées. Les types iconographiques de ces statuettes se distribuaient comme suit :

A. Contenu des niches des croix de chemins du comté de Portneuf.

Vierge

Sans spécification 10

N.-D. de Lourdes 3

Immaculée Conception 2

N.-D. du Cap

Vierge et l'Enfant

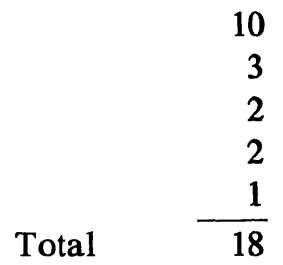

Autres

Descente de croix

6

Christ au Sacré-Cœur

3

Sainte Famille

1

Saint Christophe

1

B. Contenu des niches des croix de chemins du Lac-Saint-Jean.

Vierge

Sans spécification

N.-D. de Lourdes

5

N.-D. du Cap

4

Vierge et l'Enfant

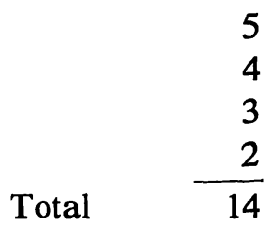

Autres

Christ au Sacré-Cour

5

Sainte Famille

Sainte Anne

(dont 2 Éducations de la Vierge)

Jean-Baptiste et Jésus

1

Images mortuaires

1

D'après les monographies paroissiales, cette fois, ce serait en premier lieu la bonne sainte Anne que les gens de Portneuf auraient le plus volontiers invoquée. Dès 1772, nous assure un 
ancien curé de la paroisse ${ }^{13}, \mathbf{M}^{\mathrm{gr}}$ Briand accordait « sur la demande du curé du Cap-Santé, conjointement avec la totalité des habitants " d'avoir sainte Anne pour seconde patronne et titulaire de l'église. Le premier patron était la Sainte Famille. C'est ensuite en 1825 que la fabrique commanda à Antoine Plamondon un tableau représentant Sainte Anne. C'est surtout, en 1913, le miracle survenu à Félixine Jacques et attribué à sainte Anne qui valut tant d'hommages à la thaumaturge de la part des auteurs de la monographie. À Saint-Raymond, par contre, c'est un forgeron que l'ivrognerie mit en contact avec le diable lui-même qui, sous la forme d'un grand cheval noir, conduisit le singulier pèlerin jusqu'à Sainte-Anne-de-Beaupré, afin qu'il demande pardon pour son intempérance ${ }^{14}$.

Toujours d'après les monographies, c'est ensuite sur l'Immaculée Conception que les gens de Portneuf auraient jeté leur dévolu. N'a-t-on pas fondé à cet effet, vers 1890, à Cap-Santé, une confrérie du Scapulaire bleu de l'Immaculée Conception, nous disent les anciens curés du lieu ${ }^{15}$. Après ces cultes prédominants à sainte Anne et à l'Immaculée Conception, viennent, loin derrière : le Sacré-Cœur, saint Joseph, la Sainte Famille et enfin saint François-Xavier, sainte Philomène et sainte Marguerite-Marie.

Au Lac Saint-Jean, c'est à la Vierge que reviendrait la première place dans le cœur de la population. On fait surtout intervenir ici la force attractive du sanctuaire du Lac Bouchette. Mais, selon $\mathrm{M}^{\mathrm{gr}}$ Victor Tremblay, la dévotion à Marie daterait au moins de l'année 1885, quand on érigea à Alma une association des Enfants de Marie «en application du mandement de $\mathbf{M}^{\mathrm{gr}}$ Racine en date du 8 mai 1880, qui recommandait le groupement des jeunes filles sous le titre d'«Enfants de Marie » et qui formulait un règlement à cet effet ${ }^{16} \%$. On fait ensuite un moindre état de la dévotion à sainte Anne qui vient cependant au deuxième rang. Vient ensuite le Sacré-Cœur puis enfin saint Joseph dont le cas est ici assez

13 Félix Gatien, "Histoire du Cap-Santé », manuscrit de 1830, publié conjointement avec ceux, plus récents, des abbés David GosseLIN et J.-Albert Fortier dans Histoire du Cap-Santé, Cap-Santé et Québec, 1955, 77.

14 Marcel LARUe, Premier centenaire de Saint-Raymond, 1842-1942, Québec, 1942.

15 Gatien, Gosselin et Fortier, op. cit., 282.

16 Victor Tremblay, Alma, au Lac Saint-Jean : son histoire, Alma, 1967, 220. 
typique. C'est d'ailleurs avec un certain humour que $\mathrm{M}^{\mathrm{gr}}$ Tremblay nous parle de "l'odyssée de saint Joseph», aux prises avec le pouvoir politique.

La triste histoire du saint patron du Canada dans cette vaste région commence en 1860 quand il doit céder sa place à « la plus vénérable des belles-mères, sainte Anne ${ }^{17}$ " comme titulaire de la colonie, sur la rive nord du Saguenay, en face de Chicoutimi. Il devient alors patron de la colonie de la Pointe-aux-Trembles (Chambord). En 1869, cependant, la visite du ministre Louis Archambault, qui apportait avec lui la promesse d'une subvention pour la construction de la chapelle, valut à saint Louis d'être préféré à l'époux de la Vierge. Selon le même scénario, deux ans plus tard, dans la colonie de Grandmont, saint Joseph doit encore céder sa place à saint Gédéon, en l'honneur de Gédéon Ouimet, ministre de l'Instruction publique. C'est enfin à Alma que le grand saint de la liturgie connaît "la fin de ses transmigrations ${ }^{18}$ ".

La comparaison de ces deux tableaux, tirés de l'analyse du contenu des niches de croix de chemins et des monographies paroissiales, nous amène à faire les constatations suivantes : à propos de Portneuf, les monographies parlent d'abord d'un culte prédominant à sainte Anne, alors que sa figuration est absente des niches des croix de chemins. En deuxième lieu, elles assurent qu'il y a eu dans ce comté un culte répandu à l'Immaculée Conception dont la figuration est en effet très abondante dans les niches. Il faut certainement assimiler les figures de Notre-Dame du Cap et de Lourdes à l'Immaculée, ce qui porte leur nombre à 7 sur un total de 18 statuettes représentant la Vierge. En dernier lieu, les monographies font état d'un culte faible ou marginal: au SacréCœur, à saint Joseph, à la Sainte Famille, à saint François Xavier ainsi qu'à sainte Philomène et sainte Marguerite-Marie, qu'il faut comparer à de rares figurations: du Sacré-Cour, de la Sainte Famille, de Saint Christophe et enfin de Saint Joseph avec l'Enfant Jésus.

A propos, cette fois, du Lac Saint-Jean, les monographies parlent d'un culte prédominant à la Vierge de l'Immaculée et nous pouvons observer dans son cas une figuration très abondante. On

17 Ibid., 99.

18 Ibid., 99-101. 
fait en deuxième lieu mention d'un culte répandu à sainte Anne et nous constatons, cette fois, contrairement au comté de Portneuf, que la sainte est bien présente au pied de quelques croix de chemins. En troisième lieu, il est question d'un culte assez répandu au Sacré-Cœur, qui est aussi fort bien représenté dans les statuettes. Finalement, les monographies locales relatent la présence de cultes faibles: à saint Joseph, qui est absent des niches, à la Sainte Famille qui en orne pourtant plusieurs; et tandis que JeanBaptiste est totalement absent des monographies, il occupe toujours sa modeste niche de la croix de la Lièvre, à Saint-Hedwidge ${ }^{19}$.

III. Peut-on nettement distinguer ici les dévotions populaires des cultes liturgiques?

Avant de donner une réponse à cette question, il convient de dresser une sorte de bilan que j'établirai en quatre points : premièrement, le fait que nous trouvons dans ces deux régions 32 des 57 niches de croix de chemins consacrées à la Vierge ne doit pas nous étonner. C'est la pratique généralisée du mois de Marie au pied des croix qui incitait les gens à choisir cette représentation plutôt qu'une autre. Il s'agit nettement ici d'un culte liturgique qui est devenu une dévotion populaire. On ne peut souhaiter plus grande unité.

Deuxièmement, la très grande corrélation qui existe entre les deux types de documents à propos du culte ou de la dévotion au Sacré-Cœur laisse supposer ici aussi une très grande unité des formes de cultes. C'est d'ailleurs au Sacré-Cour que s'était vouée une association de dames de Saint-Joseph d'Alma et qui avait dans la suite demandé à l'évêque de Chicoutimi sa reconnaissance officielle ${ }^{20}$. Une démarche en ce sens est évidemment beaucoup moins fréquente que l'inverse. Le cas de sainte Anne est plus problématique. Il y a une bonne corrélation entre les deux sources concernant son culte dans la région du Lac Saint-Jean mais ces sources se contredisent dans le cas de Portneuf, ce qui est au premier abord un peu inquiétant. Il serait pour le moins très hasardeux de conclure ici à la présence d'un culte liturgique et à l'absence de dévotion populaire à sainte Anne dans le comté de Portneuf.

19 R. JEAN, loco cit.

20 Victor Tremblay, op. cit., 124. 
Troisièmement, il faudrait plutôt expliquer l'absence de figuration de sainte Anne tout simplement par sa rareté dans le commerce de la statuaire de plâtre. Il y aurait du reste grand intérêt à interroger plus profondément le passé sur cette question, car à l'époque où ces figures étaient taillées dans le bois ou coulées en cire, il était beaucoup plus facile d'exercer un choix libre dans les sujets ${ }^{21}$. C'est donc beaucoup plus une question de marché et de type de production qui intervient ici que d'écart chronologique comme tel, car les plus vieilles statuettes peuvent dater de plusieurs dizaines d'années.

Quatrièmement, une tendance apparaît clairement à travers les deux types de sources, c'est la présence constante des grands thaumaturges qui sont l'objet de pèlerinages connus : Sainte-Annede-Beaupré, Cap-de-la-Madeleine, Lourdes, Lac-Bouchette, Montmartre. La proximité de grands pèlerinages semble donc expliquer la présence marquée de certains cultes organisés mais semble aussi déterminer le choix des statuettes, sauf toujours dans le cas de sainte Anne dans Portneuf. Ce «drainage » des lieux de pèlerinages tendrait-il donc à faire échec à des cultes indépendants?

Finalement, cette première expérience laisse supposer qu'il existe au Québec une unité beaucoup plus grande, en matière de cultes, que dans la Savoie de Van Gennep. Mais il ne faut pas conclure aussi vite, car une étape de la démarche expliquée au début a été un peu escamotée, et l'entreprendre pourrait faire toute la différence : c'est la relative pauvreté de l'enquête directe sur les dévotions auprès des propriétaires de ces croix et niches. Et voilà ce que tentera de corriger Paul Jacob dans le chapitre qui suit.

\author{
Jean Simard \\ Département d'histoire \\ Université Laval
}

21 Les voyageurs du $18^{\mathrm{e}}$ siècle : Pehr Kalm (1748), Thomas Anburey (1776) et Isaac Weld (1795) mentionnent notamment la Vierge à l'Enfant et le Christ en croix. P. Kalm, Travels in North America, New York, Dover publications, 1966 (réédition), vol. II, 416. T. ANBUREY, Journal d'un voyage fait dans l'intérieur de l'Amérique Septentrionale, Paris, 1793. I. Weld, Voyage au Canada dans les années 1795, 1796, 1797, Paris, 1803. 